\title{
Give, and You Shall Receive: Mental Health Professionals and Stigma Towards Persons with Mental Illness
}

\author{
Keng Chuan Soh* \\ Department of Psychological Medicine, Khoo Teck Puat Hospital, Singapore \\ *Corresponding author: Keng Chuan Soh, Department of Psychological Medicine, Khoo Teck Puat Hospital, Singapore, \\ Email: soh.keng.chuan@ktph.com.sg
}

\begin{abstract}
Stigma is one of the barriers that hinder people with mental illness from successfully engaging in treatment, which contributes to mental illness having a substantial global burden of disease. This review article examines the duality of the relationship between mental health professionals and stigma towards mental illness. Mental health professionals are subject to associative stigma by virtue of the people with mental illness whom they work with, which can bring about significant distress to the mental health professionals. Despite the need for a non-judgemental attitude for optimal engagement, Health care professionals are not immune from stigmatizing beliefs of their own. On top and above of anti-stigma initiatives with the general population in mind, healthcare workers have been prioritized as a target group for anti-stigma efforts. This is because they have the Potential to impact others, including both persons with mental illness and the general public, through the course of their daily work. Before their professional roles, the training stage might provide the ideal platform to introduce anti-stigma efforts. This benefits of this might not be immediately evident, but this has a long-lasting impact with the potential to turn the wheels of change at the societal level.
\end{abstract}

Keywords: Social stigma; Mental illness; Medical staff; Physician patient relations; Nursing staff; Nurse patient relations

\section{Introduction}

Persons with mental illness grapple with stigma and the discrimination that comes along with their mental health condition. At the public health level, this represents a major concern as it contributes to the treatment gap which discourages those in need of professional help from seeking assistance. Looking across the consultation table, mental health professionals themselves are also subject to associative stigma from peers who might be working in domains outside of mental health. This divide comes across as ironic, given the advocacy for an integrative approach towards mind and body. Mental health professionals work to help those with mental illness, but are individuals themselves shaped by influences and personal experience from their immediate surroundings and societal environment at large. They would also form their own opinions about people with mental illness, with some of these opinions dating back to even before undergoing training in their field.

When surveyed in comparison to the general public, mental health professionals in Australia [1] and America [2] were found to have more positive attitudes about people with mental health problems. This was also the case for Croatian medical doctors [3]. However, these results were contrary to the author's experience when surveying ward-based doctors and nurses in an acute hospital about patients with alcohol use disorder [4], which revealed stigma amongst healthcare workers to be worse than the general public. This article consolidates literature over the recent two years pertaining to stigma towards mental illness and healthcare professionals. It considers healthcare professionals from both perspectives, as the recipients of such stigma and the personnel concurrently exhibiting discriminatory attitudes.

\section{Mental Health Professionals Being Stigmatized}

Mental health professionals experience associative stigma, where they are negatively stereotyped because of the people with mental illness whom they work to help. This has an impact on them, similar to what their clients and patients would experience. While there might be a cultural component in associative stigma such that a greater degree of respect tends to be accorded to mental health 
professionals in China than the US [5], examining the manner in which Chinese psychiatrists address the topic of stigma with psychiatric patients and their relatives [6] might shed light on how this comes to be the case. As a consequence of stigma, doctors with mental health concerns might experience reluctance in opening up. Whether at the stage of a trainee [7] or beyond, disclosing mental illness might cause an individual to be perceived as "weak" and undermine their professional competence. They are hence less likely to disclose this in the work setting, to co-workers and employers alike, and to seek professional assistance for themselves. There is room for much more to be done to support professionals with mental illness. Ensuring the presence of medical care with sufficient confidentiality, occupational health support and back-towork interviews [8] are but some of the measures with potential to make a difference.

\section{Healthcare Professionals as Stigmatizers}

It is paradoxical to note that healthcare professionals concurrently exhibit stigmatizing attitudes. The "Inter Nos" (Latin for "Among Us") study surveyed attitudes of healthcare professionals towards mental illness, across multiple centres in Spain, Portugal and Italy [9]. Hospital-based staff were found to have more stigmatizing responses on both the Attribution Questionnaire (AQ-27) and The Community Attitudes towards the Mentally Ill (CAMI), agreeing more readily with restrictive options, coercion and segregation. In particular, the more discriminatory attitudes and responses tended to come from nursing assistants as well as staff in non-clinical and "other clinical" roles. When mental health professionals stigmatize the persons with mental illness whom they work with, this has the potential to undermine diagnosis, treatment and successful health outcomes [10]. Right from the start, this might adversely affect the therapeutic alliance. This in turn hinders the mental health professionals' understanding of the service user's problem, leading service users to disengage from treatment more readily [11] which ultimately sets up a vicious cycle as a self-fulfilling prophecy. This only serves to reinforce the pre-existing negative stereotypes held by the mental health professional.

\section{Education as a Possible Means of Addressing the Problem}

Before health care professionals assume their vocational roles, they are, first and foremost, individuals in society just like every member of the public. Interventions targeted at the general public would have an impact on them, but yet more can be done during the course of their professional training. Experiences at the early stages of training can certainly go a long way in terms of professional development, and others have advocated for antistigma initiatives to begin at the educational stage. Data from United States demonstrated the presence of an educational gap
[12], such that psychiatry coursework for pharmacy, nursing and social work students did not improve stigma towards mental illness. This might seem disheartening, but the identification of such educational gaps is the first step in allowing them to be addressed by means of appropriately incorporating anti-stigma training. Following exposure to the mental health curriculum, improved attitudes towards people with mental illness were found in medical students from UK [13] and South Africa [14], as well as nursing students from Turkey [15-17] There might truly be a role for the revision of curriculum pertaining to mental health, for anti-stigma programmes to be included as part of the courses. This might turn out to have a more profound educational impact than factual content found in textbooks and learnt by rote, about what constitutes mental illness and how these are treated.

\section{Future Research}

From direct contact intervention to Responding to Experienced and Anticipated Discrimination (READ), there have been no shortage of ideas on how to tackle stigma through education. However, the single most effective modality of delivering the anti-stigma message remains to be seen. Though focused on low- and middle-income countries, a recent systematic review had difficulty in pinpointing the most effective anti-stigma intervention [18]. Future head-tohead comparisons of these varying modalities would help to point the best way forward. Healthcare workers in particular have many opportunities to come into contact with persons with mental illness. For the person with mental illness, each interaction can either work out as a disappointment or a positive encounter. Their influence cannot be understated, and the impact of stigma in this group warrants anti-stigma interventions to prioritize this group. It is worthwhile to note the protocol for a randomized controlled trial in Chile [19] targeting primary healthcare workers, and to see how this would unfold.

\section{Conclusion}

Corrigan \& Nieweglowski suggested a parabolic relationship between familiarity of mental illness and public stigma [20], where people tend to stigmatize those with mental illness when they were either unfamiliar or too familiar with them. This proposal does suggest that too much of exposure might not be a good thing after all, and there exists an optimal balance in terms of the experience to deliver during training. The need to address stigma towards mental illness has never been questioned, though the best methods to do so remain elusive. While the sentiment of the public is important, this author is a believer of the saying "charity begins at home". Mental health professionals and healthcare workers working outside of mental health domains are two groups that need to be targeted. This is for the wellbeing of their service users, as well as themselves. 


\section{Financial Disclosure}

The author does not have any financial disclosure. This research has not received specific grant from any funding agency in the public, commercial or not-for-profit sectors.

\section{Declaration of Competing Interest}

The author does not have any conflict of interest.

\section{Indexing}

- Google Scholar email - caseysoh@gmail.com

- ORCID iD: https://orcid.org/0000-0002-5694-4276

\section{References}

1. Reavley NJ, Mackinnon AJ, Morgan AJ, Jorm AF (2014) Stigmatising attitudes towards people with mental disorders: a comparison of Australian health professionals with the general community. Aust N Z J Psychiatry 48(5): 433-441.

2. Stuber JP, Rocha A, Christian A, Link BG (2014) Conceptions of mental illness: attitudes of mental health professionals and the general public. Psychiatr Serv 65(4): 490-497.

3. Arbanas G, Rožman J, Bagarić Š (2019) The attitudes of medical doctors, nurses and lay people towards schizophrenia, depression and PTSD. Psychiatr Danub 31(Suppl 1): 84-91.

4. Soh KC, Lim WS, Cheang KM, Chan KL (2019) Stigma towards alcohol use disorder: Comparing healthcare workers with the general population. Gen Hosp Psychiatry 58: 39-44.

5. Lin X, Rosenheck R, Sun B, Guojun Xie, Guhua Zhong, et al. (2019) Associative stigma experienced by mental health professionals in China and the United States. Soc Psychiatry Psychiatr Epidemiol 54(6): 745753.

6. Sun KS, Lam TP, Lo TL, Wu D (2019) How Chinese psychiatrists see and manage stigmatisation of psychiatric patients: a qualitative study in Hong Kong. Evid Based Ment Health 22(2): 51-55.

7. Vally Z (2019) Mental health stigma continues to impede help-seeking and self-care efforts among trainees in mental health professions. Perspect Psychiatr Care 55(2): 161-162.

8. Grant A, Rix A, Shrewsbury D (2019) 'If you're crying this much you shouldn't be a consultant': the lived experience of UK doctors in training with mental illness. Int Rev Psychiatry 31(7-8): 673-683.

9. Del Olmo-Romero F, González-Blanco M, Sarró S (2019) Mental health professionals' attitudes towards mental illness: professional and cultural factors in the INTER NOS study. Eur Arch Psychiatry Clin Neurosci 269(3): 325-339.

10. Nyblade L, Stockton MA, Giger K, Virginia Bond, Maria L Ekstrand, et al. (2019) Stigma in health facilities: why it matters and how we can change it. BMC Med 17(1): 25.

11. Carrara BS, Ventura CAA, Bobbili SJ, Jacobina OMP, Khenti A, et al. (2019) Stigma in health professionals towards people with mental illness: An integrative review. Arch Psychiatr Nurs 33(4): 311-318.

12. Sherwood DA (2019) Healthcare curriculum influences on stigma towards mental illness: Core psychiatry course impact on pharmacy, nursing and social work student attitudes. Curr Pharm Teach Learn 11(2): 198-203.

13. Deb T, Lempp H, Bakolis I, Tushar Vince, William Waugh, et al. (2019) Responding to experienced and anticipated discrimination (READ): anti -stigma training for medical students towards patients with mental illness-study protocol for an international multisite non-randomised controlled study. BMC Med Educ 19(1): 41.

14. De Witt C, Smit I, Jordaan E, Koen L, Niehaus DJH, et al. (2019) The impact of a psychiatry clinical rotation on the attitude of South African final year medical students towards mental illness. BMC Med Educ 19(1): 114

15. İnan FŞ, Günüşen N, Duman ZÇ, Ertem MY (2019) The Impact of Mental Health Nursing Module, Clinical Practice and an Anti-Stigma Program on Nursing Students' Attitudes toward Mental Illness: A Quasi-Experimental Study. J Prof Nurs 35(3): 201-208.

16. Martínez-Martínez C, Sánchez-Martínez V, Sales Orts R, Dinca A, Richart Martínez M, et al. (2019) Effectiveness of direct contact intervention with people with mental illness to reduce stigma in nursing students. International Journal of Mental Health Nursing 28(3): 735-743.

17. Gibson R, Carson J, Houghton T (2019) Stigma towards non-suicidal self-harm: evaluating a brief educational intervention. Br J Nurs 28(5): 307-312.

18. Heim E, Henderson C, Kohrt BA, Koschorke M, Milenova M, et al. (2019) Reducing mental health-related stigma among medical and nursing students in low- and middle-income countries: a systematic review. Epidemiol Psychiatr Sci 29: e28.

19. Grandón P, Saldivia S, Vaccari P, Raul Ramirez Vielma, Víctor Victoriano, et al. (2019) An Integrative Program to Reduce Stigma in Primary Healthcare Workers Toward People with Diagnosis of Severe Mental Disorders: A Protocol for a Randomized Controlled Trial. Front Psychiatry 10:110.

20. Corrigan PW, Nieweglowski K (2019) How does familiarity impact the stigma of mental illness. Clin Psychol Rev 70: 40-50.

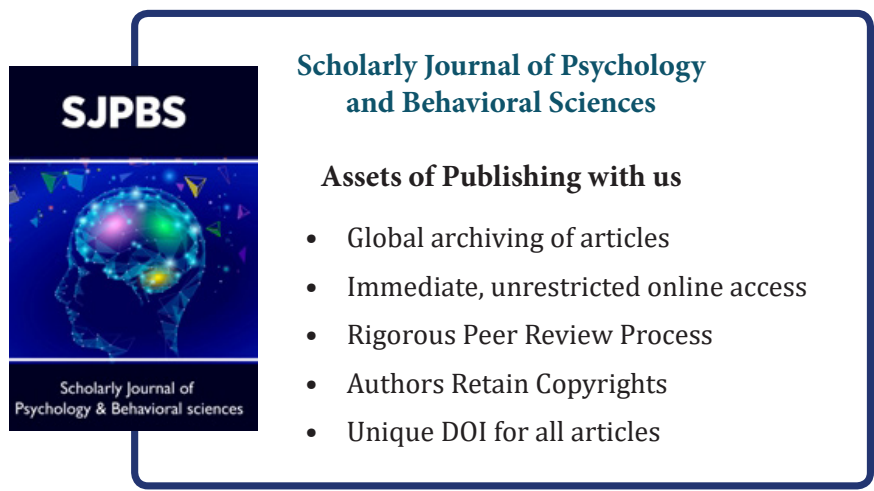

